\title{
Research into the Main Tendencies and Perspectives of Psychosocial Development of the Existing and Potential "Middle-Class" Representatives in the Modern Russian Society
}

Valeriy Aleksandrovich llyin

Tatyana Ivanovna Bonkalo

Elena Alekseevna Petrova

\author{
Vladimir Aleksandrovich Morozov
}

Oksana Ivanovna Mironova

Russian State Social University, Address: BId. 1, 4 Wilhelm Pieck Str., Moscow 129226, Russia

Doi:10.5901/mjss.2015.v6n4s2p236

\section{Abstract}

The article contains an overview of the results of research of the peculiarities of psychosocial development and the structure of psychosocial identity of typical representatives of the "middle-class", the students of the leading universities and high schools. The detailed analysis highlights the significant differences and key problems of psychosocial development of each of the three lifestages. Based on psychosocial approach, there have been analyzed the social and psychological factors for typical personal deformations and the coping mechanisms therewith.

Keywords: psychosocial development, psychosocial identity, psychosocial crisis.

\section{Introduction}

\subsection{The relevance of the research problem}

In the context of social transformations one of the key problems related to the objective need for a rather intensive innovative development of the social structure with conservation of the integrity and functionality of the society, is the problem of creation and development of the so-called "middle-class" which is a kind of backbone of modern society. At the same time, in Russian environment the process of its formation has not been painless, and the proportion of this social group within the structure of society nowadays is evidently insufficient. The reasons for such state of things were described in a number of sociological, political science, social and economical researches (Erasova, 2007, Ilyin, 2007, Michaylova, 2012, Tlostanova, 2010, Higgs and Dzhilleard, 2014). We suppose, however, it could not be understood fully without a profound investigation of peculiarities of personal and socio-psychological development of middle-class representatives in the modern Russia. This issue has become particularly relevant at the present stage of development of Russian society because, on the one hand, the tendency for increment of social activity and formation of civil society has become quite apparent, and, on the other hand, the threat of ideological and social polarization, radicalism, and extremism has aggravated, which can indeed lead to a full scale social catastrophe. (Kryukova, at al., 2014; Vinogradova, at al., 2014)

\subsection{Organization of research}

In connection with the above, we have elaborated and carried out the research into the peculiarities of psychosocial development of the three lifestages of Russian citizens, the actual and potential representatives of the "middle-class".

The hypotheses of the study was the suggestion that the structure of psychosocial identity of the three lifestage categories corresponding to the basic stages of personal and professional formation of the typical "middle-class" representatives, that is: high-school students, senior university students and the specialists of financial and consultancy enterprises with higher education, - should differ significantly. 
The empirical base of the research was compiled by a number of Moscow schools, leading humanitarian and economical universities of Moscow and Saint Petersburg, Russian financial, insurance and consultancy enterprises, as well as the audit companies.

Overall sample scope of the interviewees has amounted to 930 people, which includes representatives of the three categories: highschool students (281 people, among which -115 male and 166 female, average age — around 16 y.0.), university students, predominantly those in higher training (401 people, among which - 137 male and 264 female, average age $-20,5$ y.o.), specialists in financial sector and the organizational consultancy, with higher education (248 people, among which - 103 male and 145 female, average age of the interviewees - around 30 y.o., average experience at similar job positions at the moment of the research - around 2 years). It must be noted that the age categorization of the interviewees has been fulfilled basing on E. Erickson's periodization (Erikson, 1996), according to which the highschool students belong to the teenage developmental age, senior university students - to the earlier maturity stage and the representatives of the third category - to the maturity stage. In connection with this, we shall be referring thereon to the first and third categories of the interviewees with nominations of "teenagers" and "adults".

It should be said in this respect that the adult category of the interviewees was compiled by the specialists with higher education and working on the regular basis as middle-rank managers, organizational consultants, auditors, etc. i.e. by the typical representatives of the actual "middle-class". In its turn the interviewed students were studying precisely in the most highly-rated universities, which are generally very high valued during the evaluation of the candidates' cv for the vacancies, as was evidenced through the analysis of the empirical base of this research, i.e. of the questionnaires of adult interviewees and during the interviews with the directors of various companies and organizations. In this perspective, the three categories of the interviewees by their age and social status are reflecting the main stages of socialization of the middle class representatives, which allows us to identify the main patterns of psychosocial development in modern Russian conditions of this social group, which - as we already noted - is the key group in many ways.

\section{Methods}

The main methodology for the verification of the hypotheses of this research was the differential of psychosocial development. It has revealed the ways of resolution of the basis conflicts, relevant at the time of this investigation, of the first five stages of psychosocial development among representatives of the three lifestages: high school students, university students and adults. In addition, the investigation program has included the methods of inclusive and external supervision, and the semi-structured interview. The statistical processing of the findings was carried out via the package SPSS 17.0

Before we get to the description of the obtained results, let us mention briefly the methodology of differential of the psychosocial description (DPD) and, in particular, the substantial characteristic of the scales of differential. This methodology was elaborated and validated in $2003-2007$ 's with the purpose of identifying the dynamics of psychosocial development and structural and functional features of the psychosocial development of an individual. It features a particular semantic differential designed to reveal the results of the resolution of the basis conflicts of the development of stages of epigenetic cycle, covering the childhood and youth periods - formation of the key elements and the proper identity - and consists of the five bipolar scales: trust - mistrust; autonomy - shame, doubt; initiative - guilt; competence unsuccessfulness; identity - psychosocial confusion. In terms of the content they reflect the patterns of the respective development stages, theoretically substantiated and described according to the psychosocial concept. Detailed interpretation of the characteristics of each scale are presented in a number of available works (Gluhov, 2005; Indenbaun, 2010; Kandybovich and Sekach, 2013; Kondratiev and llyin, 2007; Stepanova, 2009), so it seems redundant to enter into it again.

\section{Results}

Comparative profiles of the five factors of differential of psychosocial development were based on calculation of average data for each category of the interviewees (Diagram 1). 


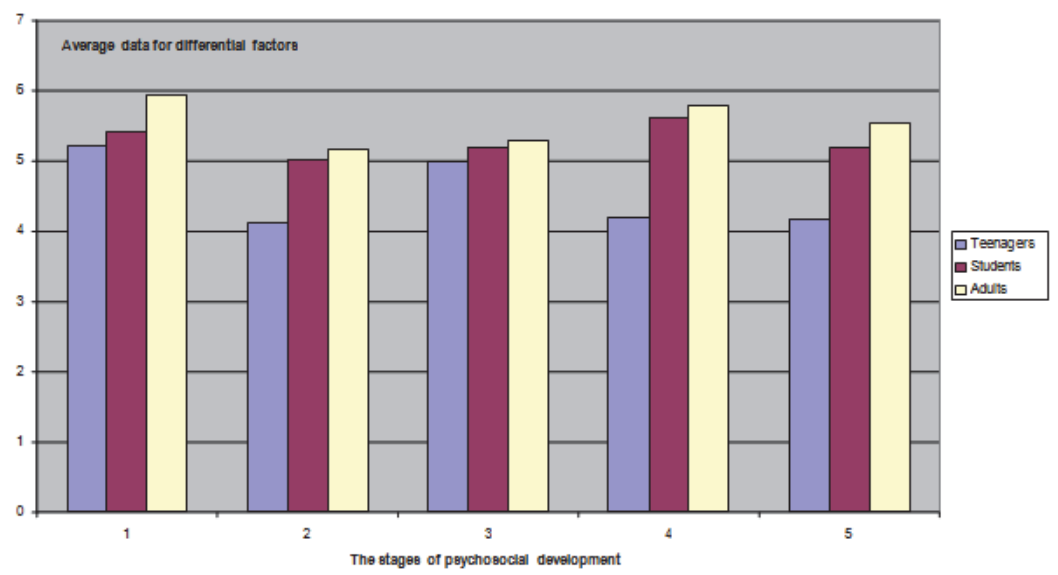

Diagram 1. Comparative profile of psychosocial development of the three age categories of the interviewees.

The dynamics of psychosocial development of students and adults follows a common trend - we can see the highest average values in both categories of the interviewees in the first (trust) and fourth (competence) factors of differential of psychosocial development. The lowest average values among these categories of the interviewees were registered in the second (autonomy) and the third (the initiative) factors of the differential, whereas the fifth (identity) factor occupies an intermediate position. With this the average values across all factors of differential of psychosocial development among the adults is higher than that of the students, and in case of the first and the fifth factors, the average difference is quite distinct.

However, the situation is fundamentally different among the teenager interviewees.

For the purpose of testing of the significance of differences seen in the Diagram, there was used the nonparametric U-test Mann-Whitney for comparison of independent samples. The results have evidenced the statistically significant differences (two-sided level of asymptotic significance of U-criteria $p<0,01$ ):

- among the teenagers and students - in the factors of autonomy, competence and identity;

— among the teenagers and adults - in all factors of differential of psychosocial development;

- among the students and adults - in the factors of trust, competence and identity. Also, in the factor of autonomy the difference is on the border of significance (two-sided level of asymptotic significance of U-criteria $p=0,011$.

Thus, it has been determined that the results of resolution of most conflicts on the basic stages of psychosocial development, which represent the main structural components of the psychosocial identity, shall differ significantly among the representatives of the three age categories.

In order to specify the structural features of psychosocial development among representatives of the three age categories of the interviewees there has been undertaken a statistical analysis of the significance of differences in the results' distribution in connection with the five factors of differential within each of the categories. With this purpose the nonparametric T-criteria Wilkoxon to compare related samples was applied. It was established that:

- for the adult category there are significant differences between the distribution of the results of the interviewees in all factors of the differential (two-sided level of asymptotic significance of T-criteria $p<0,01$ in all cases);

- for the student category there are no significant differences between the distribution of results in connection with the factors of initiative and identity (two-sided level of asymptotic significance of T-criteria $p=0,885$ ), in all other cases significant differences were recorded;

- for the teenage category there are no significant differences between the distribution of the results in connection with the factors of autonomy and competence, autonomy and identity, competence and identity (two-sided level of asymptotic significance of T-criteria amounts to 0,161, 0,370 and 0,941 accordingly), in all other cases significant differences were recorded.

This means that in our sample in general, the degree of resolution of the analyzed basis intrapersonal conflicts 
differs significantly. Thereby we have received the direct empirical confirmation of the validity of fundamental idea of heterochrony of development within the psychosocial development concept.

For the purpose of further additional specification and deep substantial interpretation of the revealed differences between and within each of the three categories of the interviewees there has been applied the analysis of the basic frequency characteristics of the empirical distributions of the received data. The Table 1 below represents the values of the basic frequency characteristics, rounded off to the second decimal place, for the factors of differential of psychosocial development in each of the three age groups, calculated through the procedure of Frequencies of SPSS package.

Table 1. The values of the basic frequency characteristics of empirical distributions obtained among the three age categories of the interviewees for the factors of differential of psychosocial development

\begin{tabular}{|c|c|c|c|c|c|c|c|c|c|c|c|c|c|c|c|}
\hline & \multicolumn{2}{|c|}{ Trust } & \multicolumn{2}{|c|}{ Autonomy } & \multicolumn{2}{|c|}{ Initiative } & \multicolumn{3}{c|}{ Competence } & \multicolumn{3}{c|}{ Identity } \\
\hline Frequency characteristics of empirical distribution & 1 & 2 & 3 & 1 & 2 & 3 & 1 & 2 & 3 & 1 & 2 & 3 & 1 & 2 & 3 \\
\hline Average & 5,28 & 5,41 & 5,95 & 4,12 & 5,08 & 5,17 & 4,99 & 5,19 & 5,30 & 4,20 & 5,62 & 5,79 & 4,17 & 5,18 & 5,55 \\
\hline Median & 5,50 & 5,57 & 6,00 & 4,00 & 5,14 & 5,14 & 5,14 & 5,14 & 5,29 & 4,00 & 5,57 & 5,93 & 4,14 & 5,29 & 5,64 \\
\hline Mode & 6,14 & 5,86 & 6,00 & 4,00 & 5,14 & 5,00 & 5,29 & 5,00 & 6,00 & 3,86 & 5,57 & 6,00 & 4,00 & 6,00 & 5,86 \\
\hline Standard deviation & 1,26 &, 82 &, 65 &, 89 &, 64 &, 61 & 1,08 &, 73 &, 64 & 1,06 &, 59 &, 55 &, 82 &, 88 &, 83 \\
\hline Minimum & 1,00 & 2,00 & 4,00 & 1,00 & 2,43 & 3,14 & 1,00 & 2,14 & 3,14 & 1,00 & 2,86 & 4,14 & 1,00 & 2,57 & 2,57 \\
\hline Maximum & 7,00 & 6,86 & 6,86 & 6,83 & 6,57 & 6,57 & 7,00 & 7,00 & 6,86 & 7,00 & 7,00 & 7,00 & 5,71 & 7,00 & 6,86 \\
\hline Percentile: 25 & 4,57 & 5,00 & 5,71 & 3,57 & 4,57 & 4,82 & 4,43 & 4,86 & 5,00 & 3,71 & 5,14 & 5,53 & 3,86 & 4,71 & 5,14 \\
\hline 50 & 5,50 & 5,57 & 6,00 & 4,00 & 5,14 & 5,14 & 5,14 & 5,14 & 5,29 & 4,00 & 5,57 & 5,93 & 4,14 & 5,29 & 5,64 \\
\hline 75 & 6,14 & 6,00 & 6,43 & 4,57 & 5,43 & 5,57 & 5,71 & 5,71 & 5,71 & 4,57 & 6,00 & 6,14 & 4,71 & 5,86 & 6,14 \\
\hline
\end{tabular}

(Note: the table below refers to numerical designations of the categories of the interviewees as follows: 1 - teenagers; 2 students; 3 - adults)

The most representative in terms of the content shall be the characteristics of the quartile shares of the obtained distributions.

As evidenced by the table above, the positive conflict resolution "trust versus mistrust" has been most clearly represented among the interviewees of the older age category.

Even the lowest quartile of distribution has been localized in the positive part of the differential scale - when transmitting the score values of minimal variable and the $25 \%$ percentile into the scale values there's an interval 0 to $+1,71$. Please be reminded that the scale value $+1,5$ is the lowest interval level which corresponds to the stable positive conflict resolution. Within this interval $(+1,5$ to $+2,5)$ there are the results of $50 \%$ of the interviewees of this category, compiling the two average quartiles - when transmitting the score values $25 \%$ and $75 \%$ percentile into the scale ones there's an interval $+1,71$ to $+2,43$. Finally, the upper quartile of distribution practically complies with the interval corresponding to the express positive resolution - when transmitting the score values into the scale ones the percentile index $75 \%$ amounts to $+2,43$, and maximal variable value $-+2,86$.

Thus, it can be stated that more than $75 \%$ of the interviewees in this category feature a stable positive resolution of the basis conflict of the first stage of psychosocial development.

The picture is rather different among the student interviewees. According to the Table, the lowest quartile of distribution of the results in this category in connection with the first factor of differential of psychosocial development complies with the scale interval from -2 to +1 (score index of minimal variable value -2 , and the $25 \%$ percentile -5 ) i.e. for the relevant part of the interviewees the basis conflict "trust versus mistrust" has either acquired negative resolution or remained unsolved at the moment of investigation. Besides, the second quartile of the distribution corresponds to the area of slightly expressed positive resolution of this conflict (scale value of $25 \%$ percentile +1 , and $50 \%-+1,57)$. Only the $50 \%$ of representatives of this category featured the stable and express positive resolution of the conflict under examination, with this the express positive resolution has been registered only with the minor part of the interviewees - the scale index of $75 \%$ percentile -+2 , and maximal variable value $-+2,86$, i.e. the higher quartile of distribution has been displaced to the scale area corresponding to the positive resolution of the conflict "trust versus mistrust".

Thus, the registered statistically relevant differences among the distribution of the results of adult and student interviewees in connection with this factor are due to the significantly higher proportion among the student environment of individuals who have never resolved or featured a slightly positive resolution of the first basis conflict of psychosocial development.

The distribution of results of the teenagers, as it was noted before, is featured firstly with the high level of 
intrapersonal differences (this factor has the maximal registered index of standard deviation $-1,26)$. The results from the lower quartile are "stretched" within all negative part of the scale and the area corresponding to a pending conflict, minimal variable index corresponds to the scale value minus 3 , and the $25 \%$ percentile $-+0,57$. The second quartile almost coincides with the area of slightly positive resolution of the conflict "trust versus mistrust", complying with the scale interval $+0,57$ to $+1,5$. Same as with the students, $50 \%$ of the teenagers feature the stable or express positive resolution of the conflict "trust versus mistrust", with this the percentage of individuals featuring the express positive resolution of this conflict is a little bit higher within this category of the interviewees - the upper quartile of distribution covers the scale interval $+2,14$ to +3 .

Thus, the results of over $50 \%$ of the interviewees of this category lie within the area of unsolved or slightly expressed positive resolution of the conflict "autonomy versus shame and doubt" - the minimal variable value corresponds to the scale index minus 0,86 , and the $50 \%$ percentile $-+1,14$. Moreover, the results of more than $25 \%$ of the interviewees also get into the area of slightly expressed positive resolution - the third quartile of distribution complies with the scale interval $+1,14$ to $+1,71$. Only the $25 \%$ of the interviewees whose results appear in the higher quartile of distribution feature the positive resolution of the conflict "autonomy versus shame and doubt" (the scale index equal to $75 \%$ percentile amounts to $+1,71$, and maximal variable value $-+2,57$ ). There were practically no individuals with the explicit resolution of this conflict in favor of autonomy within this category.

The results of the students of lower distribution quartile lie within the scale interval covering the slightly expressed negative resolution and the unsolved basis conflict of the second stage - the value of minimal variable corresponds to the point minus 1,57 , and the $25 \%$ percentile $-+0,57$. The results of another $50 \%$ of the interviewees of this category from the second and third quartiles get into the area of slightly expressed positive resolution of the conflict: scale value $25 \%$ percentile $-+0,57$, and the $75 \%-+1,43$. Only $25 \%$ of the interviewees with results from the upper quartile of distribution feature the stable positive resolution the conflict "autonomy versus shame and doubt" (scale index $75 \%$ percentile amounts to $+1,71$, and maximal variable value $-+2,57)$. Analogously to previous case, there were practically no individuals with the explicit positive resolution of this conflict.

Despite the obvious similarity of trends in psychosocial development, related to the resolution of the basis conflict of the second stage in the two categories, the proportion of negative component among the student interviewees has been evidently higher, which explains that according to the results of statistical examination the differences between distributions were found on the border of significance.

This negative trend of resolution of the basis conflict "autonomy versus shame and doubt" has been more explicit among the teenagers. The results of the $25 \%$ of interviewees of this category which have compiled the lower quartile of distribution, get into the area from the explicit negative resolution (minimal variable value corresponds to the point minus 3 ) to the lower border of the unsolved conflict (percentile index $25 \%$ corresponds to the scale value minus 0,43 ). Another almost $50 \%$ of teenage interviewees featured the unsolved basis conflict of second stage of psychosocial development as at the moment of the research - scale value $25 \%$ percentile has amounted to minus 0,43 , and $75 \%$ percentile $-+0,57$. The upper distribution quartile covers the area from mild to the explicit positive conflict resolution (maximal variable corresponds to the point $+2,83$ ).

In connection with the third factor of differential of psychosocial development, the distribution of results of adult interviewees features the following parameters.

$25 \%$ of the results from the lower quartile generally get into the scale interval corresponding to the area of the unsolved basis conflict "initiative versus guilt" (minimal variable value corresponds to the point minus 0,76 , and the index $25 \%$ percentile - point +1 ). The results of the $50 \%$ of the interviewees of this category that had got into the second and third quartiles are practically complying with the scale interval corresponding to the area of slightly expressed positive resolution of the conflict under question (scale value $25 \%$ percentile amounts to +1 , and $75 \%-+1,71$

The distribution of the results of the students features the same trend - it stands to reason that precisely in connection with the third factor of differential there were not registered any significant differences between these two categories of the interviewees. Most of the results from the lower quartile also get into the area corresponding to the unsolved basis conflict "initiative versus guilt". However, in the whole this quartile was more shifted to the negative pole of the dichotomy than in the case of the adult category (minimal variable value corresponds to the scale index minus 1,86 , and the $25 \%$ percentile - to the scale index $+0,86$ ). The results of $50 \%$ of the interviewees of this category which have compiled the average quartiles of distribution, as in previous case, are mostly covering the scale interval corresponding to the slightly expressed positive resolution of basis resolution of a conflict of the third stage of psychosocial development (scale value $25 \%$ percentile amounts to $+0,86$, and $75 \%-+1,71$ ). Finally, same as with the adult interviewees, $25 \%$ of the students with the results from the upper quartile of distribution have featured the stable of explicit positive resolution of this conflict $(75 \%$ percentile corresponds to the scale value $+1,71$, and maximal variable index - to the scale 
evaluation +3).

As for the teenage interviewees, the lower quartile of distribution of results for this factor seems stretched from the negative pole of the scale (minimal variable index corresponds to the point minus 3 ) practically towards the upper border of the scale interval corresponding to the area of unsolved basis conflict "initiative versus guilt" (scale value $25 \%$ percentile amounts to $+0,43$ ). The results of $50 \%$ of this category that had got into the average quartiles comply with the scale interval which practically coincides with the analogous distribution among the students, - the scale index $25 \%$ percentile $-+0,43$, and $75 \%-+1,71$. Diapason of the upper distribution quartile fully coincides with the analogous index for the student interviewees $-75 \%$ percentile value corresponds to the scale value $+1,71$, and maximal variable to the scale value +3 . Thus, there's the same trend in distribution of the results of the teenagers for the factor of initiative as that in the distribution of results of the students. With this the lower quartile of the distribution, compared to the adults' results, has been more shifted towards the negative pole of the scale. This explains the registered significance of differences between distributions of the results of the teenagers and adults, when there are no such differences between distributions of the results of the teenagers and students interviewees.

The results of all adult interviewees with respect to the fourth factor of differential of psychosocial development get into the positive spectrum of scale indexes, with this only a little part of the interviewees were registered with the unsolved basis conflict "competence versus unsuccessfulness" - the value of minimal variable corresponds to the scale index $+0,14$, and $25 \%$ percentile $-+1,53$. Most of the interviewees demonstrate the stable positive resolution of this conflict the results of the second and third quartiles of distribution get into the scale interval +1, 53 to $+2,14$ (value is $75 \%$ percentile). The upper quartile of the distribution of the results of adult interviewees includes the full scale interval which corresponds to the area of explicit positive resolution of the basis conflict of the fourth stage of psychosocial development - the scale index $75 \%$ percentile amounts to $+2,14$, and maximal variable -+3 .

Thus, the trend of psychosocial development of this category in connection with the fourth stage of epigenetic cycle features the high level of manifestation of the positive resolution of basis conflict "competence versus unsuccessfulness". The analogous situation, as we noticed before, takes place with respect to the first stage. However, it is noteworthy that most indexes of frequency characteristics of distribution of results have been slightly higher for the first factor, and according to the results of statistical analysis, the differences between the distributions have been significant.

The lower quartile of distribution of the results of the students for the factor of competence has been shifted towards the negative pole - scale index of minimal variable value corresponds to the point minus 1,14, and $25 \%$ percentile $-+1,14-$ i.e. it covers the area from slightly expressed negative to the slightly expressed positive resolution of the conflict "competence versus unsuccessfulness". The results of another $25 \%$ of interviewees of this category which got into the second distribution quartile, almost in whole fit into the scale interval corresponding to the slightly expressed positive resolution of the basis conflict of the fourth stage of psychosocial development (scale value $25 \%$ percentile amounts to $+1,14$, and $50 \%$ percentile $-+1,57$ ). Most of the interviewees whose results have compiled the two upper quartiles, feature the stable positive resolution of this conflict - the scale index $75 \%$ percentile amounts to +2 , and maximal variable -+3 . Please note that the comparison of frequency characteristics of distributions of results within this category for differential factors and the results of statistical examination of significance of the differences proves that precisely the basis conflict "competence versus unsuccessfulness" features the explicit positive resolution among the students.

There is a fundamentally different situation among the teenage interviewees. The results of $25 \%$ of interviewees of this category compiling the lower quartile of the distribution fit into the area from explicit negative resolution of the conflict "competence versus unsuccessfulness" (minimal variable index corresponds to the point minus 3 ) up to unsolved conflict (index $25 \%$ percentile corresponds to the scale value minus 0,29 ). Almost $50 \%$ of the interviewed teenagers feature the unsolved basis conflict of the fourth stage of psychosocial development at the time of the research - the scale index $25 \%$ percentile amounts to minus 0,29 , and $75 \%$ percentile $-+0,57$. The upper quartile of distribution covers the area from slightly to explicitly expressed positive resolution of the conflict under question (maximal variable index corresponds to the point +3 ).

It is quite understood that from the perspective of psychosocial development concept the results of the resolution of the basis conflict of fifth, integrative stage of epigenetic cycle - "identity versus psychosocial confusion" - will be of particular interest.

The results of $25 \%$ of adult interviewees fitting the lower quartile of distribution, has been spread within the scale interval covering the area from the slightly expressed negative (scale index of minimal variable value amount to minus 1,43 ) to slightly expressed positive (scale index $25 \%$ percentile amounts to $+1,14$ ) resolution of the conflict under question. The more compact second quartile has been localized at the border line of areas of slightly expressed and stable positive resolution of conflict "identity versus psychosocial confusion" - the scale value is $50 \%$ percentile - 
$+1,64$. Most of the $50 \%$ of interviewees of this category whose results have got into the two upper quartiles of distribution, feature the stable positive resolution of the basis conflict of the fifth stage (scale index of maximal variable value has amounted to $+2,86)$.

Thus, it seems clear that there is a prevailing trend to the positive resolution of conflict "identity versus psychosocial confusion" among the adult interviewees. With this the number of interviewees who featured the unsolved conflict at the moment of this research has been extremely negligible.

The situation is a bit different in case of the students' interviewees. Most part of the results registered in the first distribution quartile has been localized in the area of unsolved basis conflict of the fifth stage of psychosocial development - the amount of minimal variable value corresponds to the scale index minus 1,43 , and $25 \%$ percentile $+0,71$. The results of another $50 \%$ of the interviewees of this category that have compiled the two average quartiles of distribution, form a scale interval which in the whole corresponds to the area of slightly expressed positive resolution of the conflict "identity versus psychosocial confusion" - scale index $25 \%$ percentile $-+0,71$, and $75 \%$ percentile $-+1,86$. Finally, $25 \%$ of the students interviewees with the results fitting the upper quartile of distribution, have featured the stable or explicit positive resolution of the conflict under examination - the amount of maximal variable value corresponds to the scale index +3 .

Basing on these findings, we can acknowledge the prevailing trend towards the slightly expressed positive resolution of the basis conflict "identity versus psychosocial confusion" among the students' interviewees; however, the percentage of individuals with the unsolved conflict within this category has been considerably higher than the one in the previous category.

The lowest distribution quartile of the results of the teenagers tested happened to be obviously coerced to the negative pole of dichotomy in focus. It is stretched out in scale range covering the sphere starting from evidently negative permit till unsolved basic conflict "identity versus psychosocial confusion" (the size of the minimum meaning of a variable corresponds to scale mark minus 3 , and $25 \%$ of percentile- to scale mark minus 0,14 ). Results received by $50 \%$ of the respondents of this category made up at two average quartile of distribution keep within the scale range which on the whole corresponds to unsolved basic conflict of the fifth phase of psychosocial development (scale meaning of $25 \%$ made up to minus 0,14 , and $75 \%$ - plus 0.71 ). The majority of $25 \%$ of the teenagers tested whose results fall within the top distribution quartile has ill-defined positive solution of this conflict (scale indicator of maximum meaning of a variable is plus 1,71).

Thus, it was determined that among the teenagers tested the unsolved basic conflict of psychosocial development "identity versus psychosocial confusion" prevails what is totally natural from the psychosocial development concept point of view as the matter is exactly about chronologically vital age-related period concerning this conflict. At the same time the circumstance attracts attention that in this category of respondents the quite distinct tendency to negative solution of the conflict in focus was brought out. From the point of view of development theory it also seems totally natural against the background of the results of the teenagers tested according to the second and forth factors of a psychosocial development differential (let us remind that in this connection as the checkup with the use of Vilkonson's T-criterion the statistic-significant differences between results distribution of this category of the respondents according to autonomy, competence and identity factors lack). In fact, the question is that sufficiently serious systemic deformation of psychosocial development among large part of millennial was revealed who is currently in the fifth stage of epigenetic cycle, which can have a great effect on the state of psychosocial balance in Russian society. If we compare the results by the factor of three categories of identity of the subjects, it is easy to see clear dynamic: the unresolved basis crisis of the fifth stage of psychosocial development to adolescents - poorly marked a positive resolution to the students - sustainable positive resolution to adults against practically the same level of inter-individual differences of the results by a factor at all three categories (the mean of the standard deviation was $0.82,0.88$ and 0.83 , respectively). According to this research, we get direct empirical evidence that, as a rule, the formation of psychosocial identity of the individual is not completed by 20 years and continues to the next developmental psychosocial development in modern Russian conditions.

\section{Discussion}

The comparison of the results from the three categories for the trust factor allows us highlighting various basic regularities.

Firstly, it should be noted that the clearly expressed positive resolution of the first basis conflict of psychosocial development is characteristic for the adult group. The negative trend for resolution of this conflict features mostly the teenage group. The students hold an intermediary position therein. This enables us, firstly, to come to the conclusion that in connection with our sample of interviewees there is a general positive dynamics of psychosocial development in terms 
of resolution of the first basic crisis.

Secondly, we have therefore obtained the empirical confirmation of the theoretical principles of psychosocial development concept, according to which the results of the resolution of the basis conflict of a certain stage can be substantially corrected at the individual level in the course of further development.

Thirdly, the documented evidence of reduction of intrapersonal differences of the interviewees from teenagers to adults allows us to conclude that the representatives of the same generation forming the same social group during the process of psychosocial development experience the alignment of the individual results of the resolution of the first basis conflict.

In connection with the second factor of differential of psychosocial development, the highest indexes of basic values of central trend were obtained during the distribution of the results of the adult interviewees. However, the spread of distribution through the quartile shares differs significantly from the previous case.

Frequency analysis of the distribution of the results of the three categories of interviewees in connection with the second factor of the differential of psychosocial development has revealed the general conservation of patterns registered for the first factor. This means that the process of alignment of results of resolution of the second crisis of psychosocial development within the same generation is a) chronologically more compact, and b) shifted to earlier stages of epigenetic cycle - by the moment of transition from adolescence to early adulthood it shall be basically completed.

We would like to point out that the second stage of psychosocial development is traditionally considered the most problematic one in the Russian society, which by far is stipulated by the specifics of social institutions of politics and law. We can find out the analogous conclusions with the other researchers. Thus, in particular, L.D. Gudkov notes: "The basic configuration of Russian features in the perception by Russians of themselves - it is a complex of particularistic set of characteristics with the passive authoritarian complex of dependence and subordination. These definitions compiles the image of Russians "for themselves" - passive, patient, simple (not pretending for a higher level of requests, autonomy and self-sufficiency, the complexity of basic set of values)" (Gudkov, 2004) With this, L.D. Gudkov believes that "autoqualification as "simple, open-hearted" - is not just a sign of social and cultural "qualityless". It expressed the lack of any demands for the autonomy, group or status inherent value and self-sufficiency".

The conclusions of L.D. Gudkov seem especially relevant in this context due to the fact that his own determination of identity, although being explicitly sociological, represents fully the system-forming ideas in the interpretation of identity from the point of psychosocial development concept, that is: correlations of individual and social development and procurement of the integrity and subjectness of an individual in the changing social environment.

In this perspective the applied logical correlation for the interpretation of results for the first differential factor: "positive resolution of the basis crisis of psychosocial development - social success" also constitutes an important interpretational key. On the one hand, according to psychosocial development theory the consistent progressive social mobility of an individual would just be impossible in case of the negative resolution of the basis intrapersonal conflict of the second stage of psychosocial development and formation of a stable self-alienation in the form of pathological selfidentity. In the modern Russian environment, it is especially true in connection with the high-professional intellectual activities in those sectors sufficiently integrated into the world economy. At the same time, in the context of conservation (and, moreover, propagation) of the strong paternalist traditions within the society and the existence in all levels of social regulation of the informal normative systems, the activity of the individuals with a high level of personal autonomy is often being interpreted as a threat towards the existing status quo and harshly obstructed. In such situation the most adequate way for the achievement of average level of social success would be precisely the slightly expressed positive - "rather autonomy than shame and doubt" - resolution of the basis conflict of the second stage (which has been registered with the most adult interviewees in our investigation). The individuals who featured the explicit positive, or, on the contrary, the negative resolution of this conflict in this situation have - incredible as it may seem - practically equal chances to achieve the super-success - to get to the high-end social groups (its specific variety which exists in the contemporary Russian society) or to gradually marginalize.

The third stage of psychosocial development is placed distinctively in an intermediary position between the first and the second stages. All three categories feature the statistically significant differences between the distribution of results for the factors of initiative and trust, as well as for the factors of initiative and autonomy.

According to the frequency analysis of distributions, the basic regularities defined through the examination of the results in connection with the first factor of differential of psychosocial development, shall be true in connection with the factor of initiative, and in the latter case they shall be expressed more explicitly than in the case of the autonomy factor.

When applying the same interpretational key "positive resolution of the basic intrapersonal crisis - social success" to the obtained data, the intermediary position of the third stage of the psychosocial development becomes easily understandable. In the terms of professional activities directly connected with the highly competitive information economy, 
the initiative and the sense of purpose are the objectively necessary requirements for the individual success. At the same time, the abovementioned peculiarities of the social practices established in the Russian society shall inevitably contradict to such necessity. As a result, the "excessive" personal initiative shall be "absorbed" by the system both at the stage of taking of the decision and in the process of its implementation, due to the deeply rooted practices such as innumerous approvals, endorsements, quotations, presenting of reports etc. Under these conditions the most adequate way to get into the "middle class" would be the slightly expressed positive resolution of the basis conflict "initiative against the sense of guilt", same as in case of the conflict of second stage of psychosocial development. We believe the main difference in this context shall be the fact that in the modern Russian environment such resolution of the second basis conflict would in fact be necessary, when in case of the third basis conflict it shall be just the sufficient precondition to obtain the average level of social success.

Thus, the main trend of psychosocial development of the teenagers with respect to the fourth stage of epigenetic cycle coincides almost entirely with the trend defined in connection with the second stage (there are no statistically significant differences between the distributions of the results of the interviewees for relevant factors of the differential), and it is characterized by the prevailing unsolved basis intrapersonal conflict "competence versus unsuccessfulness" with rather explicit negative pole of this dichotomy. This fact is quite alarming, because such trajectory of psychosocial development creates a real threat of the escalation among the youth of antisocial personal attitudes, commitment to the destructive religious and ideological doctrines, membership in the informal groups of criminal orientation etc. It has reason behind it that at the present day we can observe the growth of juvenile crime with the general background of increasing aggressiveness among the young people.

One of the main reasons of the detected deformation of psychosocial development becomes clear if we take into account that the sensitive lifetime - from the point of resolution of the basis conflict "competence versus unsuccessfulness" - of most of the interviewed teenagers has coincided with the boundary of 1990's and 2000's, when Russian secondary education, and elementary school in particular, was suffering the severe crisis. In connection with this we would like to mention that, according to the obtained findings, within the following years when our interviewees were studying in secondary and high school, and up to the present moment there has been no radical correction of the results of resolution of the basis conflict of fourth stage of epigenetic cycle. We believe it is due to the fact that during the reformation of the secondary school the main attention was paid to the organizational aspects and the content of education. At the same time, the most important, from our point of view, developmental function of the school remains on the periphery of the educational process.

The level of intrapersonal differences in distribution of the results of adults and students interviewees almost coincides. There is the similar situation with respect to the second factor of differential of psychosocial development. At the same time the level of intrapersonal differences in distribution of the results of the teenagers with respect to this factor has been notably higher, than in case of the factor of autonomy. We suppose it can be explained by the fact that in this case it is not the dynamics of alignment of the results of resolution of the basis crisis of psychosocial development among representatives of the same generation that is concerned, but the intrapersonal criterion of social selection at the moment of entering a university. In other words, it is precisely the competence, and personal competence, that could not be reduced to a standard set of "knowledge and skills", that shall be the determinant from the point of successfulness of an individual who's entering a university, especially in case of the prestigious academic institutions giving a quality education. As for the substantive differences of the results in connection with the factor of competence, the algorithm "the success creates the success" is clearly operative in the scheme specified in the course of the analysis of the results received for the first factor of the differential of psychosocial development.

If you try to identify the principle which caused this kind of hypogenesis in the Russian condition, then, according to psychosocial development concept, the most significant ones are represented by two points.

Firstly, the institution of ideology in Russia for a number of reasons is completely unable to meet the «universal psychological drive for a system of ideas that give a convincing picture of the world» (Bonkalo et al., 2014; Feldstein, 1995; Solodova, 2008; Stepanova, 2009) in an accessible and attractive to young people's consciousness form.

In another words, representatives of the younger generations are mostly deprived of necessary comprehensible and universal guidelines related to solving personal problems on the considered stage of development "through a free role experimentation to find their niche in society, a niche that is firmly defined and it corresponds to him exactly" (Kondratiev, M.Y. and Ilyin, 2007) Explicit failure and, moreover, defectiveness, the point of solution of this problem, the results of the efforts of modern Russian ideologues - from propaganda clichés about "EMERGING knees" to pseudoscientific euphemism "sovereign democracy" - appear to be self-evident.

This inefficiency in the present context of the existing social system is being compensated at the individual level through local kvaziideologies produced by the informal group membership, organizational culture of universities, 
corporations and the like, as well as individual system of values of certain high-referential representatives of the social environment and personal experience. It is obvious that the formation of individual values by selection in such type of semistructured and usually collisional environment almost inevitable delays permit intrapersonal level of the basic conflict "identity versus psychosocial confusion."

Secondly, in modern Russian conditions the young people in the majority are deprived of the possibility of the full stay psychosocial moratorium. This is caused by the fact that the society doesn't provide necessary delay at this stage of development, in other words "time-out", in relation to the individual performance of civil and professional functions. The most odious manifestation of this kind is a continuing compulsory universal military obligation to age of 18 years. Certainly, in this context we are able to speak about of the rules of electoral law, tax, labor, civil codes. Along with this, there are a number of destructive, in terms of psychosocial moratorium, rooted in real social practice moments, not only officially codified, but also formally banned. For example, one of the major problems associated with the quality of higher education is that many full-time undergraduates are forced to work according to the scheme of full employment, often in the areas that are not directly related to their professional specialization.

For example, one of the major problems connected with quality of higher education is that many full-time students have to work full day, often in areas that are not directly related to their professional specialization. At the same time, due to a number of reasons, both psychological and socio-economic nature, the process of young people separation in modern Russian conditions is unreasonably prolonged. Therefore, after entering the University (of course, if it is not associated with a move to another city) a significant proportion of young people continues to live with their parents. Moreover, educational and psychotherapeutical practices show that even for students who at first sight leading free lifestyle, in majority of prosperous families (from a traditional point of view) there is a high degree of parental control throughout the study at the university.

In general, organization of higher education is demonstrative in terms of psychosocial moratorium. Most part of American and Western European institutions of higher education carries on the tradition of midlevel's universities, which with their actual autonomy and internal regulations served in many ways as a kind of "social incubators", where the younger generation had the opportunity to live out the age liminality, almost without being under pressure and regulatory restrictions adopted in the broad society. At most Russian universities, students do not have this opportunity.

Obviously that lack of possibility to live out psychosocial moratorium in chronologically age-to-date period leads to the conservation of the status of personal liminality ("liminality" in social psychology is defined as "intermediate, in a sense, a transitional social situation of the person in the system of social relations, characterized by the loss of the former social status and associated status-role position in a non-entry into a new social role "( Huhlaeva, 2002, Levin and Zakharenkov, 2013, Mansurov, 2014, Mikhailova, 2012, Safronov, 2013, Safronov, 2008), which, in turn, prevents the resolution of the underlying conflict "identity versus psychosocial confusion."

Results of the completed research in features of psychosocial development at the basic stages of representative of three age categories fully confirmed validity of this hypothesis. In addition, they revealed a number of regularities of the middle class formation in modern Russia in the context of individual psychosocial development, as well as some of the typical problems associated with the psychosocial development of the younger generation, and their underlying causes.

\section{References}

Bonkalo, T.I., Petrova, E.A., Korolev, L.M., Babich, E.G. \& Karpov, E.B. (2014). Psychological factors of destructiveness in formation of personal national identity. Asian Social Science,10, 24, 278-284

Erasova, N.Y. (2007). Becoming a professional identity of the individual in the context of the psychosocial approach to development. Psychological Science and Education, 5, 111-118

Erikson, E. (1996). Identity: Youth and Crisis. - M .: Progress

Feldstein, D.I. (1995). Social psychological patterns of personality development in ontogenesis. Questions of psychology, 6, 23-46

Gluhov, V.P. (2005). Fundamentals of psycholinguistics - M .: AST

Gudkov, L.D. (2004). Negative identity. - M .: New Literary Review

Higgs, P., Dzhilleard, K. \& Jones, Y.R. (2014). Selfish generation: a study of the concept. Sociology of power, 3, 10-30.

Huhlaeva, O.V. (2002). Developmental Psychology: youth, maturity, old age. - M.

Ilyin, V.A. (2007). Using a psychosocial approach to the study of social and psychological processes in modern society. Questions psychology, 2, 109-123.

Kandybovich, S.L. \& Sekach, M.F. (2013). Human mental stability of human capital, 9 (57), 159-166.

Kondratiev, M.Y. \& Ilyin, V.A. (2007). The ABCs of Social psychologist practice. - M .: PER SE

Levin, I.L. \& Zakharenkov, V.V. (2013). Criteria, indicators and levels of mental health of children and adolescents. International Research Journal, 7-5 (14), 43-44

Mansurov, S.E. (2014). Integrity as a system property of human existence. European Social Science Journal, 5-1, 9-16. 
Mikhailova, E.A. (2012). A comparative study of the structure of psychosocial identity of various social and age categories of modern Russians [electronic resource]. Psychological Science and Education psyedu.ru.,1. URL: http://psyjournals.ru/psyedu_ ru/2012/n1/50853.shtml (date accessed: 20/02/2015)

Safronov, M.V. (2013).Psychosocial well-being of students: risk and protective factors. - Novosibirsk

Solodova, T.V. (2008). Psychosocial study of students. Bulletin of the Russian State Pedagogical University, 60, 468-473.

Stepanova, L.G. (2009). Formation of gender identity in modern boys and girls. Psychological Science and Education, 5, 67-72

Tlostanova, M.V. (2010). The man in the modern world: problems of multiple identities. Questions of social theory, 4, 191-217

Kryukova, E.M., Vetrova, E.A., Maloletko, A.N., Kaurova, O.V., Dusenko, S.V. (2014) Social-economic problems of Russian monotowns. Asian Social Science, T. 11. № 1. C. 258-267.

Vinogradova, M.V., Kryukova, E.M., Kulyamina, O.S., Vapnyarskaya, O.I., Sokolova, A.P. (2014) Approaches to the study of the status and trends of drug abuse, rehabilitation and reintegration of drug users . Biosciences Biotechnology Research Asia,Vol. 11(3), $1505-1514$ 